\title{
Máquina virtual de remachado textil
}

\section{Virtual machine of riveted textile}

\author{
CAMARENA-LÓPEZ, Stephany Samayrani†*, CASTRO-LEÓN, José Manuel, VEGA-TOLEDO, \\ José Jesús y GONZÁLEZ-DURÁN, José Eli Eduardo
}

Instituto Tecnológico del Sur de Guanajuato

ID $1^{\mathrm{er}}$ Autor: Stephany Samayrani, Camarena-López / ORC ID: 0000-0002-7139-2561, CVU CONACYT ID: 977524

ID $1{ }^{\mathrm{er}}$ Coautor: José Manuel, Castro-León / ORC ID: 0000-0003-3020-2175, CVU CONACYT ID: 977580

ID $2^{\mathrm{do}}$ Coautor: José Jesús, Vega-Toledo / ORC ID: 0000-0002-5276-3435, CVU CONACYT ID: 1000484

ID $3^{\text {er }}$ Coautor: José Eli Eduardo, González-Durán / ORC ID: 0000-0002-6897-9716, CVU CONACYT ID: 331544

DOI: $10.35429 / J I E .2019 .9 .3 .1 .9$

Recibido 04 de Julio, 2019, Aceptado, 03 de Septiembre, 2019

\begin{abstract}
Resumen
Uno de los procesos en la industria textil es el ensamblaje de ojillos en los distintos productos, para ello se utiliza una "Remachadora". Esta máquina se utiliza para colocar remaches sobre prendas textiles o de calzado, para fines estéticos o de refuerzo. Existen tres tipos de Ojilladoras: "Manuales", "Semiautomáticas" y "Automáticas"; uno de los problemas que estas presentan es la calibración manual puesto que se requiere intervención humana. Se desarrolló una máquina virtual de una remachadora textil automática utilizando como software CAD a SolidWorks dónde se crea el diseño de los sistemas mecánicos que integran a la máquina virtual, en conjunto se utiliza un software de programación visual, LabVIEW, en el cual se desarrolla un panel frontal, que es capaz de controlar todo el proceso de producción siempre y cuando exista un enlace entre estas dos interfaces mediante "LabVIEW Soft Motion". La comunicación entre estos dos softwares logra caracterizar una Máquina Virtual de Remachado Textil dónde se mejora en la realización del remachado de ojillos en menos de dos segundos, la capacidad de remachar 4 tamaños diferentes de ojillos y la calibración automática mediante un sistema mecánico denominado LongWorthChuck, lo que contribuye a una optimización de las remachadoras convencionales.
\end{abstract}

Maquinaria virtual, Proceso de remachado de ojillos, LongWorth-Chuck

\begin{abstract}
One of the process in the textile industry is the eyelet assembly in the different products, for it is used a "Riveter". This machine is used to place rivets on textile garments or footwear, for esthetic or reinforcement purposes. There are three types of riveters: "Manuals", "Semi-automatic" and "Automatic"; one of the problems that these presents are the manual calibration because human intervention is required. A virtual machine for an automatic textile riveter was developed using CAD software as SolidWorks where the design of the mechanical systems that integrate the virtual machine, together a visual programming software, LabVIEW, is used, in which a front panel is developed, capable of controlling the entire production process as long as there is a link between these two interfaces through "LabVIEW Soft Motion " The communication between this two software achieve to characterize a "Virtual Machine of Riveted Textile" where it is improved in the riveting of eyelets in less than two seconds, the ability to rivet 4 different sizes of eyelets and automatic calibration using a mechanical system called "LongWorth-Chuck", this contributes to an optimization of conventional riveting machines.
\end{abstract}

Virtual Machinery, Eyelet Riveting Process, LongWorth-Chuck

\footnotetext{
* Correspondencia del Autor (Correo electrónico: zam.camarena@ gmail.com)

$\dagger$ Investigador contribuyendo como primer autor.
} 


\section{Introducción}

En la zona sur del estado de Guanajuato como principal fuente de ingresos es la producción textil o procesos del mismo giro, DENUE (Directorio de Estadístico Nacional de Unidades Económicas -Inegi) revela 1,449 unidades económicas en fabricación de prendas de vestir entre Moroleón y Uriangato; normalmente la mayoría de sus procesos se encuentran automatizados; pues se debe cumplir con la demanda del producto durante las diferentes temporadas del año. Algunos de sus principales procesos se muestran en la figura 1, y en la mayoría se encuentra la automatización presente con el objetivo de facilitar y agilizar dichos procesos, uno de esos procesos en particular es el remachado de ojillos. Constituyendo la colocación de dos piezas metálicas: ojillo macho y ojillo hembra, coloquialmente llamadas "ojillo" y "contra", figura 2, sobre una prenda siendo fusionadas mediante una fuerza física logrando un ensamble, figura 3, para brindar una mejor presentación a los productos, en los últimos años se ha hecho más presente en el sector textil, lo cual indica un nivel de automatización bajo o intermedio.

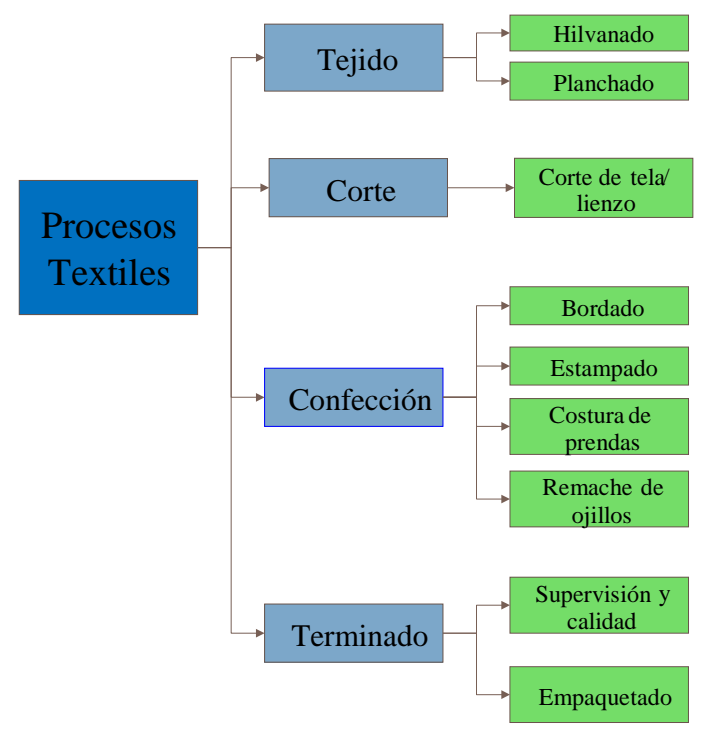

Figura 1 Procesos dentro de la industria textil

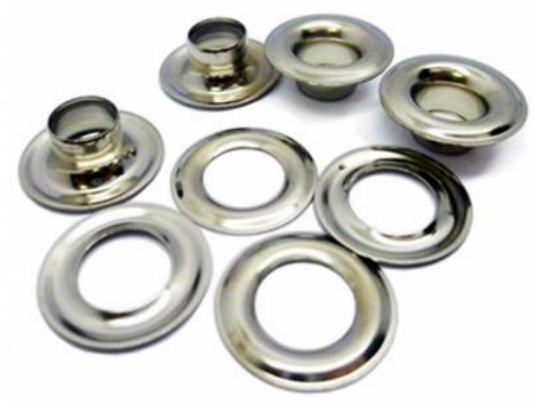

Figura 2 Ojillos machos y ojillos hembra

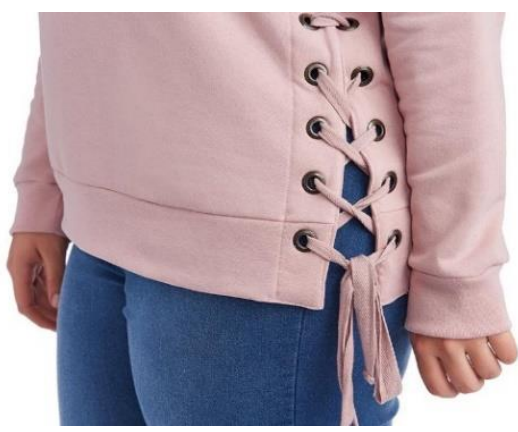

Figura 3 Remaches textiles en prenda de vestir

Actualmente, la mayoría de remachadoras son manuales, figura 4 , y en el mejor de los casos son semiautomáticas; es necesario el soporte de un operador para manipularlas y trabajarlas, son bastante similares en cuanto a características técnicas y principio de funcionamiento ya sean eléctricas o neumáticas; lo que las difiere es la flexibilidad que tiene cada una de ellas para trabajar con diferentes tipos de ojillos y tiempos de remaches por minuto.

Existe una máquina remachadora semiautomática VD-403 de la marca AVANCE®, figura 5. Donde el acomodo de las contras y sus respectivos ojillos son de manera manual; además cuando se desea cambiar de tamaño de ojillo es necesario quitar piezas de la máquina o bien, el mecanismo de dados, figura 4, lo que implica pérdidas de tiempo en las calibraciones de estas y solo es capaz de remachar 3 ojillos de diferentes tamaños: 1in, 3 in $\mathrm{y} 5 \mathrm{in}$.

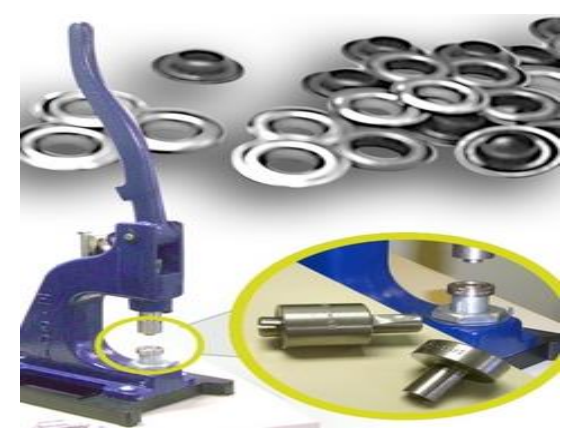

Figura 4 Remachadora textil manual con mecanismo de dados.

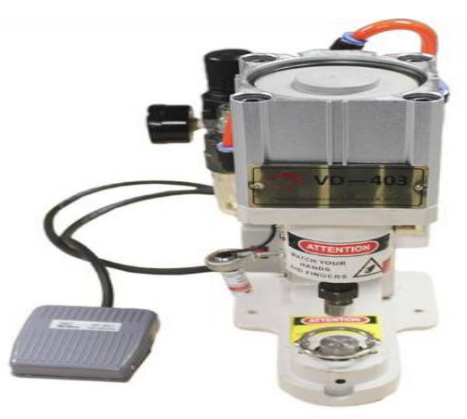

Figura 5 Remachadora textil semiautomática VD-403 de AVANCE ${ }^{\circledR}$

CAMARENA-LÓPEZ, Stephany Samayrani, CASTRO-LEÓN, José Manuel, VEGA-TOLEDO, José Jesús y GONZÁLEZ-DURÁN, José Eli Eduardo. Máquina virtual de remachado textil. Revista de Ingeniería Industrial. 2019 
En otras maquinarias el acomodo de ojillos es automático figura 6 y figura 7, pero la fuente que brinda la fuerza necesaria para completar un remache es un motor eléctrico, el cual se encuentra en constante funcionamiento y requiere de un arranque para poder realizar un remache.

Por la naturaleza de los motores cuando se arranca un motor se consume un pico de corriente mientras se estabiliza dicha máquina, pero al generar constantes arranques provoca que se presenten más picos de corriente, lo que a su vez implica un mayor consumo de energía representante de un gran gasto adicional.

Observando también que algunas de estas remachadoras automáticas solo son capaces de remachas un solo tamaño de ojillo, mientras que las otras requieren de aditamentos especiales para cambiar el tamaño de ojillo lo que genera otra inversión.

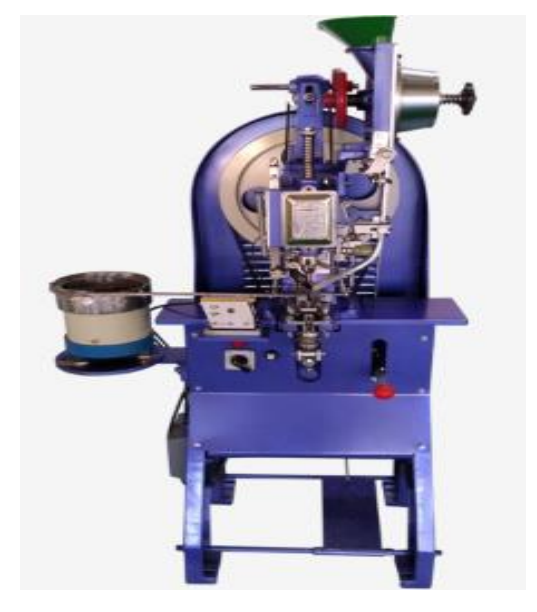

Figura 6 Máquina remachadora textil automática LMCCHA1 DE LEWMACO®

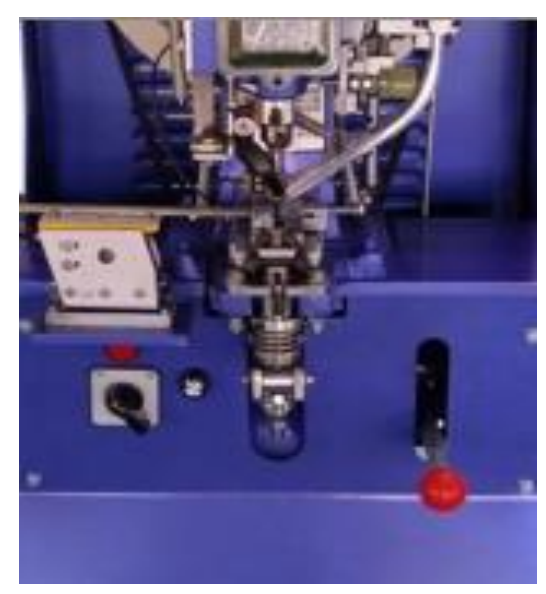

Figura 7 Mecanismo automático para remache de ojillo macho y ojillo hembra
Para reducir los costos en el diseño de maquinaria, y tener un mejor rendimiento en producción, actualmente se están implementando nuevas herramientas para el diseño de maquinarias en donde se hace uso de softwares de diseño asistido por computadora permitiendo hacer modelados en 3D como lo es SolidWorks; una herramienta tan eficaz que permite realizar estudios de movimiento de los componentes del diseño, también permite realizar comunicaciones con otros softwares como lo es LabVIEW; una interfaz de programación visual y además la ejecución de sus instrucciones son de manera paralela, permitiendo una ejecución más rápida del programa para el control de los movimientos de los componentes de SolidWorks y ambos programas en conjunto conforman una máquina virtual mediante el enlace SoftMotion; para el análisis de los diseños de maquinarias y permitiendo una visión más específica del comportamiento real de la máquina sin necesidad de invertir mucho dinero y evitando las pruebas destructivas.

Haciendo uso de las nuevas tecnologías y del nuevo método de diseño de maquinaria como se ilustra en la figura 8 , para desarrollar una nueva propuesta de remachadora textil, con su modelado en SolidWorks y su control en LabVIEW todo interconectado mediante el módulo LabVIEW SoftMotion.

Para demostrar sus funcionalidad como propuesta para la industria textil del sur de Guanajuato, en el presente trabajo se muestra el procedimiento para completar esta serie de pasos para llegar a un fin común, la máquina virtual de remachado textil .

\section{Metodología a desarrollar}

Teniendo el objetivo de automatizar un proceso de producción ejecutado en una máquina virtual, se establece un proceso representado esquemáticamente por 7 etapas; la figura 8 muestra secuencialmente cada uno de los métodos que se implementaron para diseñar la maquinaria virtual. 


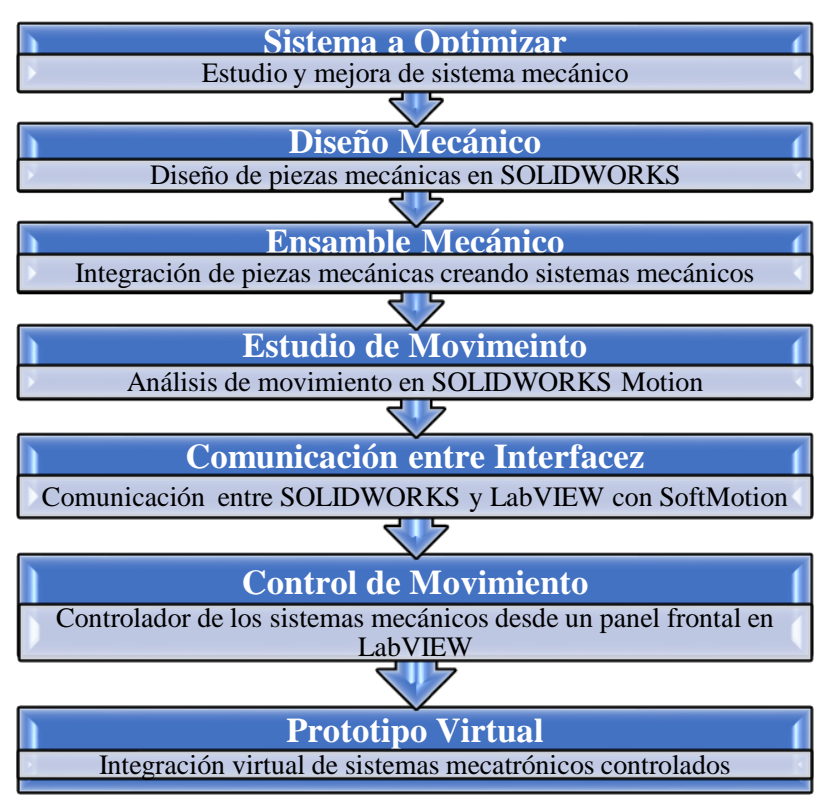

Figura 8 Proceso para el diseño de maquinaria virtual.

\section{Sistema a optimizar}

En la industria textil-calzado se realiza un proceso que requiere de un remache de ojillos, en el cual se detecta que para este ensamble se requiere de intervención humana por la necesaria calibración de los dados y el posicionamiento de los ojillos dentro de los mismos. Dónde se da por hecho que el mecanismo de los dados, figura 4, es el causante de una producción ineficiente. Se propone un mecanismo llamado "LongWorth Chuck" para la sujeción de ojillos macho, figura 9 , y unas canaletas ajustables, figura 10, para administración de ojillos macho y ojillos hembra.

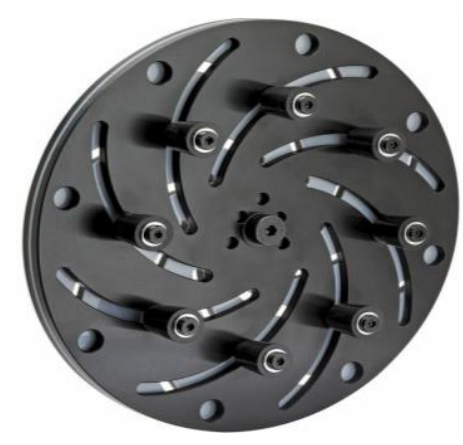

Figura 9 LongWorth Chuck

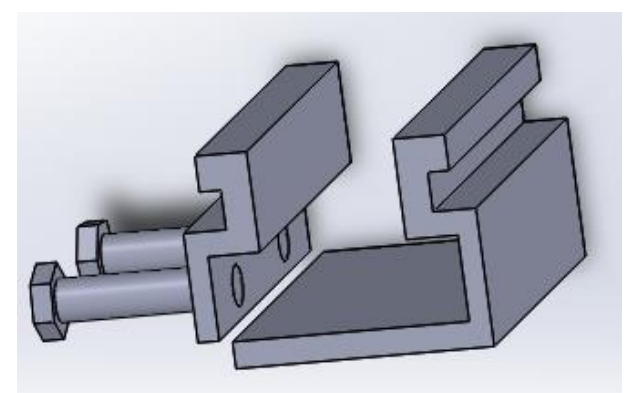

Figura 10 Propuesta de las canaletas ajustables

\section{Diseño Mecánico}

Una vez identificado el sistema a optimizar se procede a realizar su diseño mecánico dentro de SolidWorks, figura 11 y figura 12, comenzando con el diseño de piezas, las cuales cumplen su objetivo de realizar operaciones dentro del proceso del remachado de ojillos una vez que estas son integradas en un ensamble mecánico.

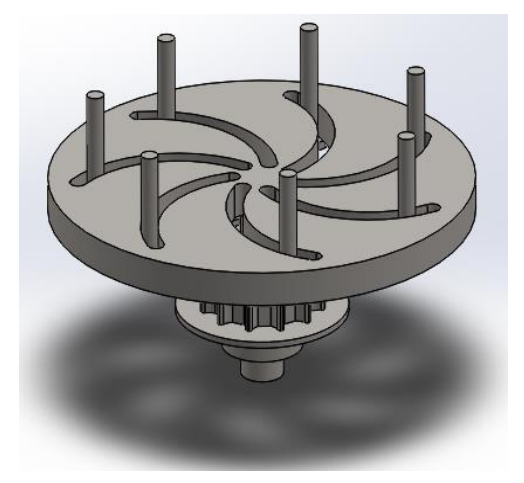

Figura 11 Diseño del LongWorth Chuck

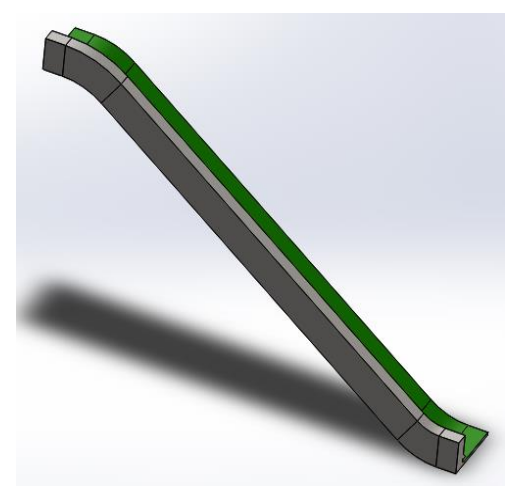

Figura 12 Diseño de la canaleta ajustable

\section{Ensamble Mecánico}

Terminadas las piezas se derivan los ensambles mecánicos, dónde cada una de las piezas diseñadas cumplen como un elemento dentro de un ensamble, estructurando así diferentes sistemas mecánicos, los cuales son longorth chuck, canaletas, contenedor de contras, contenedor de ojillos, sujeción de contras (pirámide) y pistón neumático, esto para verificar las dimensiones que se plantearon dentro de las piezas mecánicas, para visualizar el cumplimiento del área de trabajo y el material utilizado en el proceso de remacho de ojillos.

Posteriormente estos mecanismos se utilizan como subensambles, para conformar la remachadora virtual, figura 21 . 


\section{Estudio de Movimiento}

Teniendo los sistemas mecánicos con las dimensiones adecuadas, se procede a realizar un estudio de movimiento que nos permite visualizar el comportamiento físico de toda la integración mecánica, es decir, se obtiene información clara y exacta de las respuestas que tienen los sistemas al someterse a movimientos lineales y rotacionales específicos. Estos se realizan desde la interfaz de SolidWorks para tener una perspectiva más detalla de los escenarios equivocados o apropiados que nos proporciona el diseño de la incorporación de los sistemas.

\section{Comunicación entre interfaces}

Al comprobar que no existen colisiones o algún otro inconveniente en el diseño, se realiza una comunicación entre dos tipos de software: LabVIEW y SolidWorks, este enlace es llamado NI LabVIEW SoftMotion, figura 13. Dónde se crea un proyecto dentro de LabVIEW y se agrega cada uno de los motores que se desean controlar, ya sean lineales o rotacionales. Al tener el diseño en una interfaz y el control en otra evita la saturación de recursos en algún software, teniendo respuestas más rápidas y eficientes. Permitiendo crear un controlador más eficaz y completo al igual que un diseño más definido.

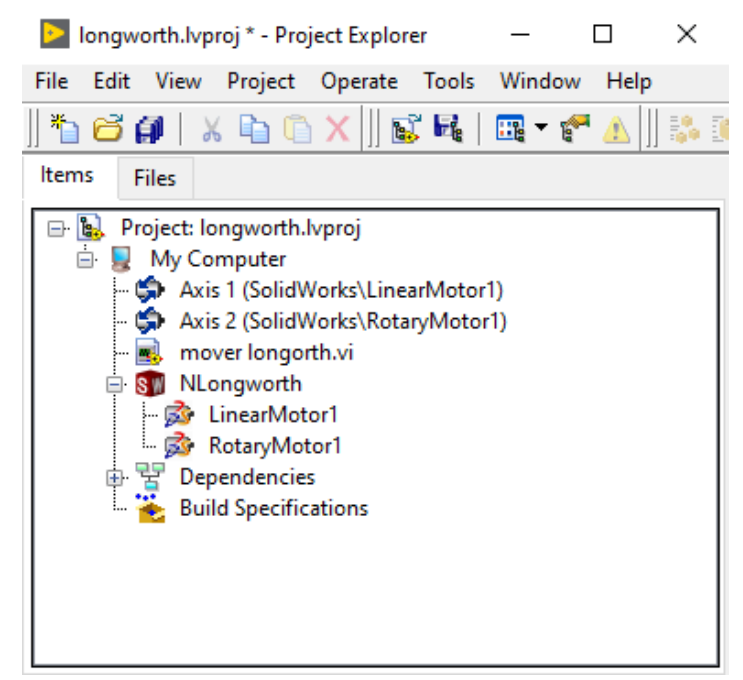

Figura 13 Comunicación entre SolidWorks y NI LabVIEW

\section{Control de movimiento}

Al concretar el enlace se tiene la capacidad de crear un control de movimiento desde un proyecto en LabVIEW para operar el diseño de la máquina remachadora en SolidWorks.
Este proyecto está compuesto por la integración del software, SolidWorks con los respectivos diseños mecánicos realizados para la remachadora virtual, el controlador (diagrama de bloques) y el panel frontal en LabVIEW, figura 14. Esto nos ayuda a tener indicadores y controles para visualizar y operar directamente el movimiento del diseño en tiempo real.

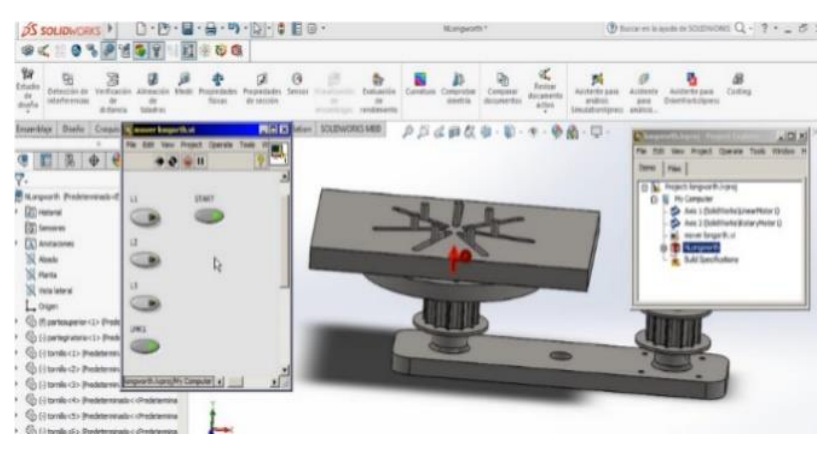

Figura 14 Integración de SolidWorks y LabVIEW.

\section{Prototipo Virtual}

El control de movimiento se estructura para cada uno de los sistemas mecánicos que conforman todo el diseño de la máquina virtual, con las especificaciones respectivas. Para así tener el control de las operaciones que debe realizar cada pieza, subensamble y ensamble que componen al diseño, figura 22 , formando así una integración virtual de sistemas mecatrónicos controlados, en otros términos, una máquina virtual de remachado textil.

\section{Resultados}

Al término del diseño, se pueden observar todos los mecanismos que se diseñaron integrados en el prototipo virtual de la remachadora, con todo el análisis previo en SolidWorks Motion, se decidió verificar el movimiento de los mecanismos más destacados por la innovación y su modelo de utilidad proporcionado en el prototipo remachadora virtual, con el enlace LabVIEW SoftMotion se tendrá mayor fiabilidad en los rangos de movimiento de los mecanismos y garantizar que se pueden tener las especificaciones requeridas. Los mecanismos sometidos a mayor estudio fueron: LongWorth Chuck, canaletas ajustables y las pirámides.

\section{LongWorth Chuck}

El funcionamiento del LongWorth Chuck es el mecanismo que ayuda en la sujeción de los cuatro distintos tamaños de ojillo: $29 \mathrm{~mm}$ (L1), $23 \mathrm{~mm}$ (L2), $20 \mathrm{~mm}$ (L3) y $15 \mathrm{~mm}$ (LMK1) respectivamente.

CAMARENA-LÓPEZ, Stephany Samayrani, CASTRO-LEÓN, José Manuel, VEGA-TOLEDO, José Jesús y GONZÁLEZ-DURÁN, José Eli Eduardo. Máquina virtual de remachado textil. Revista de Ingeniería Industrial. 2019 
Para el control se implementó un Instrumento Virtual (VI) en donde se visualiza un botón de inicio y otros cuatro botones para poder seleccionar con cual tipo de ojillo se pretende trabajar, al estar activado el inicio y algún otro botón del tamaño del ojillo se ajusta al tamaño del ojillo seleccionado.

En la figura 15, se muestra el rango de movimiento requerido para poder sujetar el ojillo con el perímetro exterior más pequeño también se observa el VI de control con la activación para remachar el ojillo más pequeño (LMK1).

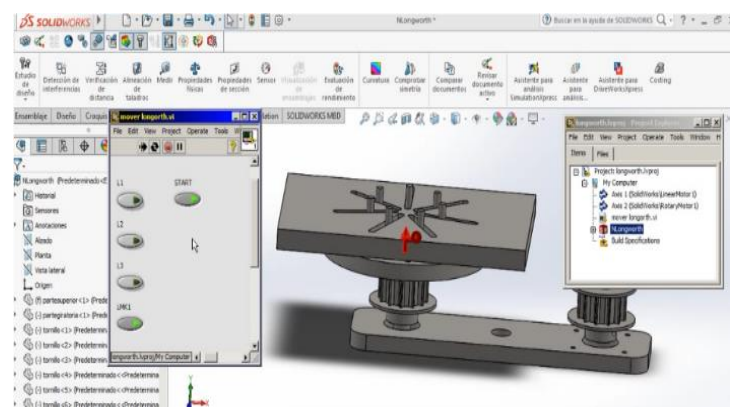

Figura 15 Rango de movimiento necesario para sujetar el ojillo LMK1

\section{Canaleta}

Se mantienen los mismos rangos en el tamaño de los ojillos, L1, L2, L3 y LMK1, donde la canaleta se ajusta al tamaño de los ojillos, el VI de control para el movimiento es idéntico al requerido en el LongWorth Chuck utilizando dos botones para hacer funcionar el control, figura 16.

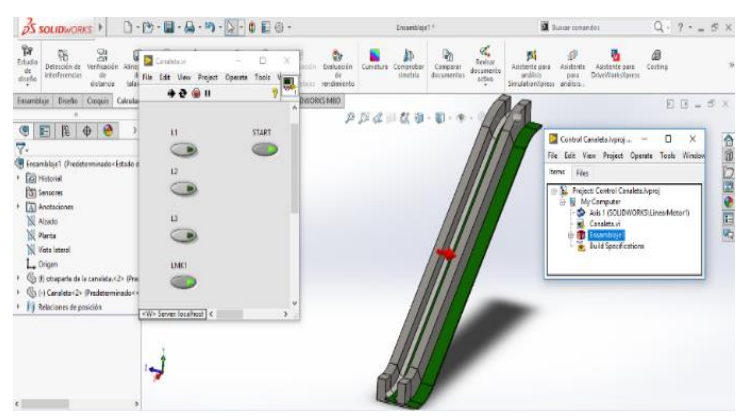

Figura 16 Rango de movimiento necesario para el ajuste de la canaleta para el ojillo LMK1

El último mecanismo analizado fueron las pirámides, las cuales nos ayudaran al posicionamiento correcto del remache para que el ojillo y la contra sean ensamblados correctamente.

\section{Pirámides}

Se denominó pirámide inversa, figura 17 , al mecanismo a donde llegará la contra, para poder ser remachada con el ojillo. Al tener las pirámides con los diámetros exactos de cada uno de los ojillos a trabajar, se garantiza que, al momento de aplicar la fuerza, la contra estará centrada y embonará con el ojillo presente y en sujeción por el LongWorth Chuk; la pirámide inversa está compuesta de 2 partes, esto con el propósito de que se puedan separar al momento de ya estar el ojillo listo para someterse a presión. Para ejercer la presión y seguir manteniendo la posición de la contra se utiliza la pirámide, figura 18, manteniendo la contra en un diámetro exacto también presente en la pirámide.

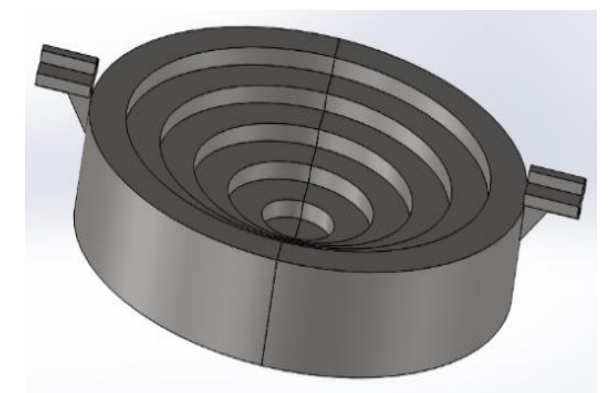

Figura 17 Pirámide Inversa

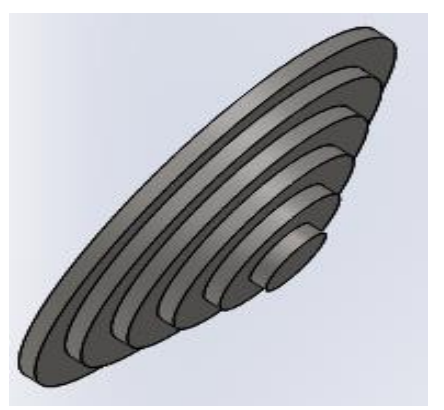

Figura 18 Pirámide

$\mathrm{Al}$ igual que los mecanismos anteriores, se hizo un VI de control para poder observar el comportamiento de este mecanismo. Se puede observar en la figura 19 que tanto como la pirámide inversa y la pirámide están en su posición inicial, esto porque no se tiene presionado ningún botón en el panel frontal.

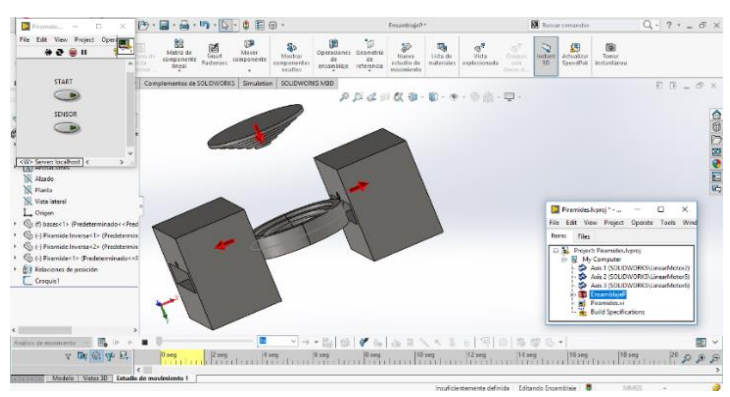

Figura 19 Posición inicial de las pirámides

CAMARENA-LÓPEZ, Stephany Samayrani, CASTRO-LEÓN, José Manuel, VEGA-TOLEDO, José Jesús y GONZÁLEZ-DURÁN, José Eli Eduardo. Máquina virtual de remachado textil. Revista de Ingeniería Industrial. 2019 
Al presionar el botón de START que se encuentra en el panel frontal, la pirámide se comienza a desplazar de manera lineal y en dirección hacia abajo como se muestra en la Figura 20.

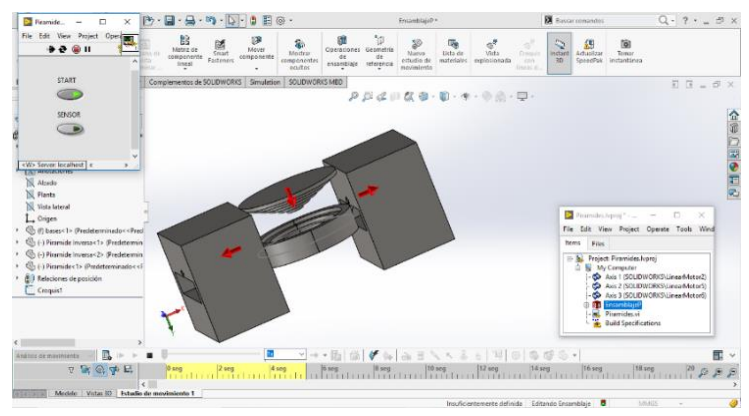

Figura 20 Desplazamiento de la pirámide

La pirámide se estará desplazando, habrá un momento en su desplazamiento que ocurra una interferencia entre la pirámide y la pirámide inversa, en este punto se simuló dentro del VI un sensor, que nos ayudara a saber que ya ocurrió la interferencia y la pirámide inversa hará un desplazamiento lineal y lateral para ceder el paso a la pirámide y pueda realizarse el remache de los ojillos, esto se puede observar en la figura 21 .

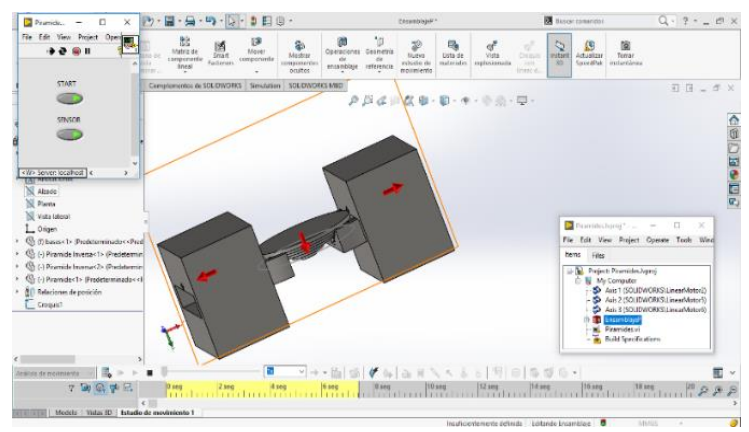

Figura 21 Activación del sensor y desplazamiento de las 2 partes de la pirámide inversa.

Logrando controlar cada uno de los sistemas mecánicos e integrarlos, se conforma la máquina virtual de remachado textil mostrada en la figura 22.

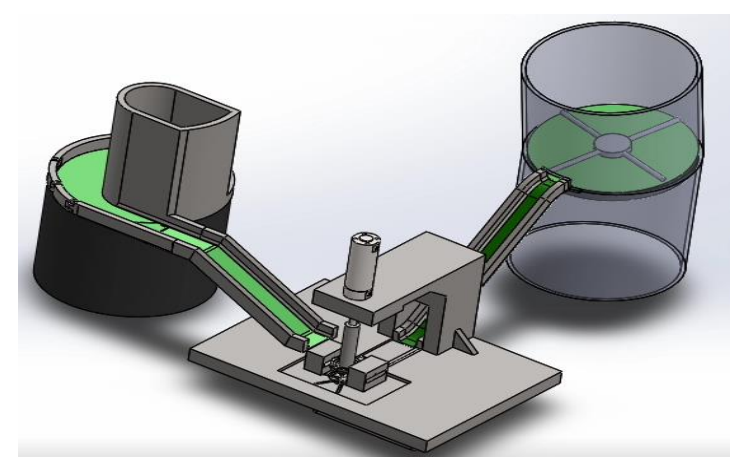

Figura 22 Máquina Virtual de Remachado Textil
En la figura 23 se observa cada uno de los mecanismos integrados de la máquina virtual de remachado textil.

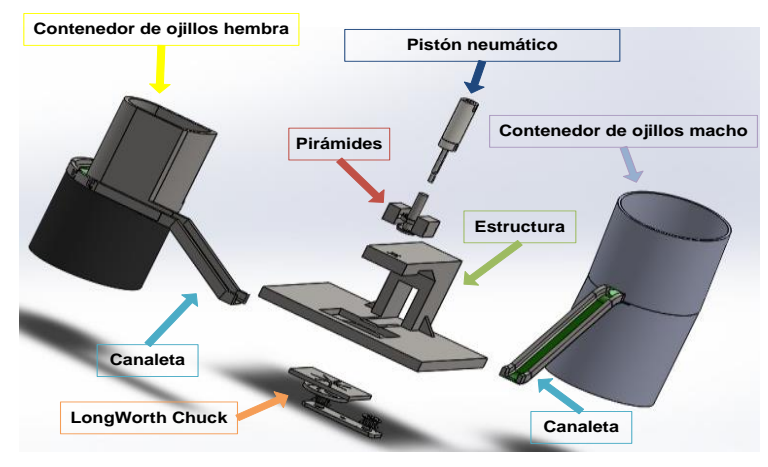

Figura 23 Mecanismos de la remachadora.

El remachado del ojillo se comienza llenando los contenedores, uno con los ojillos y el otro con las contras, los contenedores tienen un movimiento rotacional para hacer mover las piezas y logren deslizarse por las canaletas; las canaletas tienen un movimiento lineal para ajustarse al tamaño del ojillo seleccionado al igual que al LongWorth, al estar posicionado y sujetado el ojillo y la contra el pistón comienza a bajar para realizar la unión de las piezas.

Las medidas al finalizar el diseño de la remachadora son las que se muestran en la figura 24.

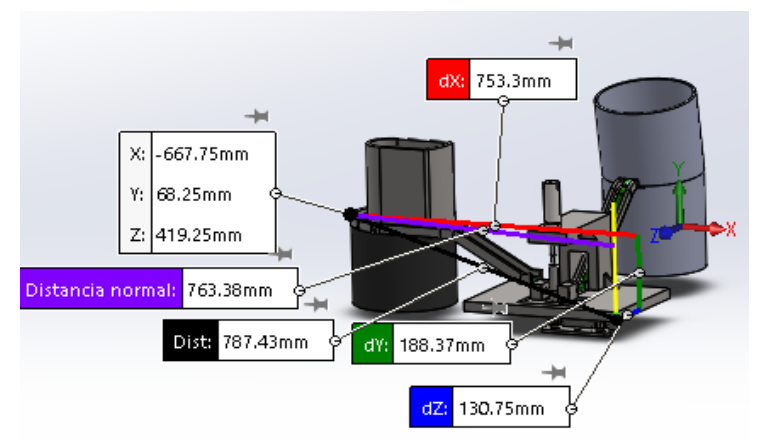

Figura 24 Dimensiones de la remachadora

Se tiene el análisis de peso y volumen teniendo en cuenta como material el acero 1020, esto se puede apreciar en la figura 25 .

Propiedades de masa de Ojilladora00
$\quad$ Configuración: Predeterminado
Sistema de coordenadas: -- predeterminado --
Masa $=79601.71$ gramos
Volumen $=11305798.69$ milímetros cúbicos
Área de superficie $=2224379.67$ milímetros cuadrados

Figura 25 Propiedades de masa la remachadora 


\section{Conclusiones}

Se hace un análisis de la competencia y se desarrolla la tabla 1 , dónde a la máquina virtual de se denomina DMVRT-04 y pertenece a la empresa DOXCRO®.

Se realiza un presupuesto maestro para la producción de 10 máquinas remachadoras con maquinado en tornos CNC y fresadoras $\mathrm{CNC}$, figura 26.

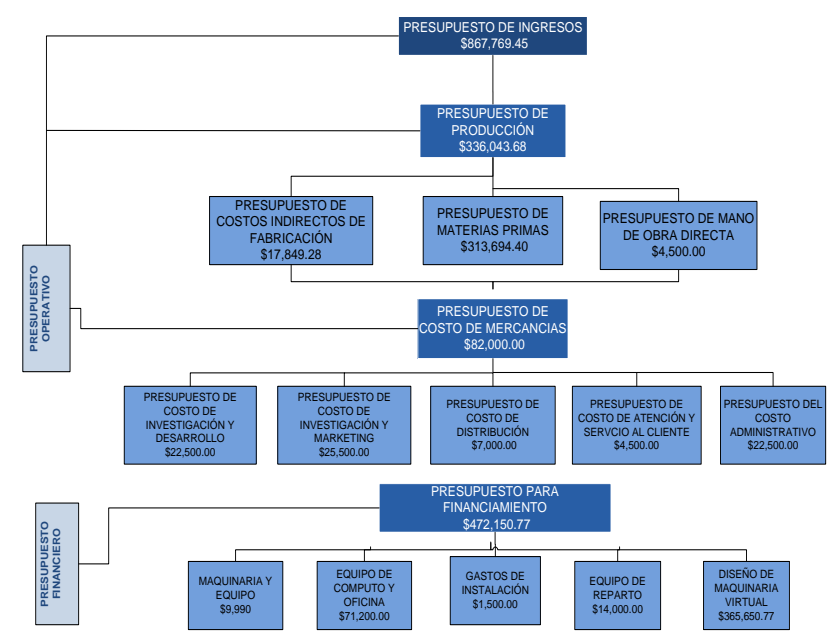

Figura 26 Presupuesto maestro con producción en maquinado

\begin{tabular}{|c|c|c|c|}
\hline $\begin{array}{l}\text { Modelo/ } \\
\text { Características }\end{array}$ & VD-403 & $\begin{array}{l}\text { LMC- } \\
\text { CHA1 }\end{array}$ & DVMRT-04 \\
\hline Empresa & Avance ${ }^{\circledR}$ & Lee-maco ${ }^{\circledR}$ & Doxcro® \\
\hline Ojillos & 3 & 2 & 4 \\
\hline Aditamientos & $\mathbf{S i}$ & $\mathbf{S i}$ & N.A \\
\hline $\begin{array}{ll}\text { Costo } & \text { de } \\
\text { inversión }\end{array}$ & $\$ 17,292+$ Dados & $\begin{array}{l}\mathbf{\$ 1 0 4 , 0 0 0} \\
+\$ 8,000 \\
\text { aditamentos }\end{array}$ & N.A \\
\hline Garantía & 1 año & 3 meses & $\begin{array}{l}\text { Contrato } \\
\text { vigente }\end{array}$ \\
\hline Arrendamiento & No & No & $\$ 2,500$ \\
\hline Capacitación & No & $\begin{array}{ll}\text { Gratis los } \\
\text { primeros } & 3 \\
\text { meses. } & \\
\end{array}$ & $\begin{array}{l}\text { Sin costo en } \\
\text { cada } \\
\text { actualización }\end{array}$ \\
\hline Mantenimiento & No & $\begin{array}{l}\text { Servicio } \\
\mathbf{\$ 4 5 0}\end{array}$ & $\begin{array}{l}\text { Sin costo en } \\
\text { cada servicio }\end{array}$ \\
\hline Actualización & No & No & Disponibilidad \\
\hline Calibración & Manual & Manual & Automática \\
\hline Proceso & Semiautomático & Automático & Automático \\
\hline Dimensiones & $\begin{array}{ll}\text { Alto: } & 33 \mathrm{~cm} \\
\text { Ancho: } & 18 \mathrm{~cm} \\
\text { Largo: } & \\
25 \mathrm{~cm} & \end{array}$ & $\begin{array}{l}\text { Alto: } 145 \mathrm{~cm} \\
\text { Ancho: } \\
85 \mathrm{~cm} \\
\text { Largo: } \\
\text { 50cm } \\
\end{array}$ & $\begin{array}{ll}\text { Alto: } & 75 \mathrm{~cm} \\
\text { Ancho: } & 75 \mathrm{~cm} \\
\text { Largo: } & \\
48 \mathrm{~cm} & \end{array}$ \\
\hline
\end{tabular}

Tabla 1 Características de la competencia.

Lo que nos deja un costo unitario de $\$ 86,776.945$ pesos mexicanos. La máquina se pretende rentar como un producto/servicio con las siguientes suscripciones:

\footnotetext{
- $\quad$ Plan anual- $\$ 60,000.00$

- $\quad$ Plan bienal- $\$ 85,200.00$

- $\quad$ Plan trianual- $\$ 90,000.00$
}

Sugiriendo contratar la suscripción trianual $\mathrm{y}$ así se obtiene una máquina remachadora textil con tecnología de punta con un arrendamiento de $\$ 2,500$ mensuales durante tres años, incluyendo capacitaciones, actualizaciones y mantenimiento preventivo.

El propósito principal fue el diseñar la máquina virtual de remachado textil, para ello se cumplieron los objetivos, comenzando con tener un diseño virtual en un programa de CAD para posteriormente analizar su movimiento en simulación, se desarrolló su control para manejar los movimientos a como se es deseado; esto para evitar los desperdicios al realizar prototipos de la forma tradicional con el método de prueba y error, el siguiente paso a realizar ya con la máquina virtual y observando que se obtuvo un comportamiento deseado es el imprimir en 3D las piezas mecánicas diseñadas para hacer el prototipo físico, por último, es crear, diseñar e implementar el control del prototipo físico al igual que el prototipo virtual, con la diferencia de pretender usar microcontroladores para reducir costos en materia prima y logré ser un producto rentable para los usuarios.

\section{Agradecimientos}

Se agradece la disponibilidad de los docentes de Ingeniería Electrónica del Instituto Tecnológico Superior del Sur de Guanajuato por el apoyo al proyecto, disponibilidad para resolución de dudas, asesoramiento sobre el diseño, delegaciones para aprender a usar el control y la proyección para la realización y culminación de la primera etapa del proyecto.

\section{- $\quad$ Dr. Fuentes Hernández Carlos Alberto \\ - $\quad$ MC. Ortega Alejos Julio \\ - $\quad$ MC. Estrada Rojo Leonel}

\section{Referencias}

Avance Imagina Crea Innova. (n.d.). Retrieved from

https://www.avanceytec.com.mx/equipos/ojilla dora-neumatica-vd-403/

Celaya, T. d. (s.f.). Programación. Obtenido de Recuperado de http://webcache.googleusercontent.com/search? $\mathrm{q}=$ cache:6DfJE2biMj0J:www.iqcelaya.itc. $\mathrm{mx} / \sim$ vicente/Programacion/Lenguajes.doc $+\& \mathrm{~cd}=1 \&$ $\mathrm{hl}=\mathrm{es} \& \mathrm{ct}=\mathrm{clnk} \& \mathrm{gl}=\mathrm{mx}$ 
Díaz, S. G. (s.f.). Diseño y control en tiempo real de un brazo de robot mediante una interfaz virtual. Obtenido de Recuperado de https://www.ecorfan.org/bolivia/researchjournal s/Tecnologia_e_innovacion/vol3num9/Revista_ Tecnologia_e_Innovacion_V3_N9_16_6.pdf

INEGI. (s.f.). Obtenido de https://www.inegi.org.mx/app/mapa/denue/

Instrumentation, N. (Segundo Trimestre de 2009). La Evolución de LabVIEW: Decadas de Desarrollo. Obtenido de Recuperado de https://docplayer.es/5723878-Instrumentationla-evolucion-de-labview-decadas-de-desarrollopagina-3-newsletter-ni-com.es.html

Instruments, N. (s.f.). Entorno NI LabVIEW. Obtenido de Recuperado de http://www.ni.com/academic/students/learnlabv iew/esa/environment.htm

Instruments, N. (s.f.). Juegos de herramientas NI LabVIEW. Obtenido de Recuperado de http://sine.ni.com/nips/cds/view/p/lang/es/nid/1 0447

Instruments, N. (s.f.). Modulo LabVIEW SoftMotion. Obtenido de Recuperado de http://sine.ni.com/nips/cds/view/p/lang/es/nid/1 4234

Introducción a la programación. (s.f.). Obtenido de Recuperado de http://dis.unal.edu.co/ programacion/book/mod ulo1.pdf

Lajara Vizcaino, J. R. (2007). LabVIEW Entorno Gráfico de Programación. ALFAOMEGA GRUPO EDITOR, S.A.

LEEMACO. (n.d.). Retrieved from http://leemaco.com/categorias/estoperol-ypedreria/productos/maquina-de-chaton-rivet

Rojas, L. O. (s.f.). Diseño asistido por computador. Recuperado de https://lsi.ugr.es/ cad/teoria/Tema1/RESUMEN TEMA1.PDF.

SOLIDBI. (s.f.). Obtenido de Recuperado de https://solid-bi.es/solidworks/

Warren J. Luzadder, J. M. (2000). Fundamentos de Dibujo en Ingeniería. 